\title{
Michel parameters in radiative muon decay
}

\author{
A.B. Arbuzov ${ }^{a, b, 1}$ and T.V. Kopylova ${ }^{b}$ \\ ${ }^{a}$ Bogoliubov Laboratory of Theoretical Physics, Joint Institute for Nuclear Reasearch, \\ Joliot-Curies str. 6, Dubna, Russia \\ ${ }^{b}$ Department of Higher Mathematics, Dubna State University, \\ Universitetskaya str. 19, Dubna, Russia \\ E-mail: arbuzov@theor.jinr.ru, tv-kopylova@mail.ru
}

ABSTRACT: Radiative muon and tau lepton decays are described within the model-independent approach with the help of generalized Michel parameters. The exact dependence on charged lepton masses is taken into account. The results are relevant for modern and future experiments on muon and tau lepton decays.

KEYwords: Beyond Standard Model, Effective field theories

ArXIV EPRINT: 1605.06612

\footnotetext{
${ }^{1}$ Corresponding author.
} 


\section{Contents}

1 Introduction 1

2 Preliminaries and notation $\quad 1$

3 Radiative muon decay 2

4 Conclusions 4

A Functions $F, G$, and $H$

$\begin{array}{ll}\text { B Generalized Michel parameters } & 6\end{array}$

\section{Introduction}

Studies of muon decay are one of the keystones in particle physics. This decay is almost a pure weak-interaction process. That allows us to use it for high-precision tests of the Standard Model (SM) and searches for new physics, see e.g. the review [1].

According to the principle of lepton universality that is adapted in the SM, the pure leptonic modes of tau lepton decays are described by the same Feynman diagrams as the corresponding muon decays. The only difference is coming from the fact that the masses of charged leptons are changed. As concerning models beyond the SM, some of them predict violation of lepton universality for tau leptons, assuming that the third generation is more strongly coupled to some "new physics" than the first two ones $[2,3]$. This motivates experimental searches for new physics in tau decays at various high-energy machines including the LHC, see e.g. $[4,5]$. In particular, a new method to probe magnetic and electric dipole moments of tau lepton using precise measurements of the differential rates of radiative leptonic decays at high-luminosity $B$-factories was proposed in [6]. Note that a high precision has been already reached in measurements of radiative leptonic tau decays at the $B$-factories, see e.g. [7].

The precision of the Michel parameters [8-10] definition from tau decays [11-15] is not yet competing with the one achieved in muon decays. Nevertheless, high statistics of tau lepton observation at the $B$-factories Belle and BaBar, and at the LHC provides good perspectives for studies of various tau lepton decay modes.

\section{Preliminaries and notation}

Within the SM, muon decays are described by interactions of vector currents formed by left fermions. Meanwhile, many models beyond the SM predict contributions of other kinds. Since the energy scale of new physics is (most likely) higher than the electroweak scale, 
the corresponding contributions can be parameterized by contact four-fermion interactions with different currents and coupling constants. The matrix element of the muon decay can be presented in the general form [16]

$$
\mathcal{M}=4 \frac{G_{0}}{\sqrt{2}} \sum_{\substack{\gamma=\mathrm{S}, \mathrm{V}, \mathrm{T} \\ \epsilon, \omega=\mathrm{R}, \mathrm{L}}} g_{\epsilon \omega}^{\gamma}\left\langle\bar{l}_{\epsilon}\left|\Gamma^{\gamma}\right| \nu_{l}\right\rangle\left\langle\bar{\nu}_{\tau}\left|\Gamma_{\gamma}\right| \tau_{\omega}\right\rangle
$$

see for details the Particle Data Group review [17] and references therein. Here index $\gamma$ denotes the type of the interaction: scalar $(\mathrm{S})$, vector $(\mathrm{V})$ or tensor $(\mathrm{T})$; and $\Gamma^{\gamma}$ are $4 \times 4$ matrices defined in terms of the Dirac matrices:

$$
\Gamma^{\mathrm{S}}=1, \quad \Gamma^{\mathrm{V}}=\gamma^{\mu}, \quad \Gamma^{\mathrm{T}}=\frac{1}{\sqrt{2}} \sigma^{\mu \nu}=\frac{i}{2 \sqrt{2}}\left(\gamma^{\mu} \gamma^{\nu}-\gamma^{\nu} \gamma^{\mu}\right)
$$

The indices $\omega$ and $\epsilon$ denote the chiralities of the initial and final charged leptons, respectively. For a given pair $(\omega, \epsilon)$ the chiralities of neutrinos are uniquely determined. Tensor interactions can contribute only for opposite chiralities of the charged leptons. This leads to the existence of 10 complex coupling constants, $g_{\epsilon \omega}^{\gamma}$. The Standard Model predicts $g_{L L}^{V}=1$ and all others being zero. Choosing the arbitrary phase by defining $g_{L L}^{V}$ to be real and positive leaves 19 real numbers to be determined by the experiment. As long as one is interested in the relative strengths of the couplings, it is convenient to require the following normalization condition:

$$
\begin{aligned}
N \equiv & \frac{1}{4}\left(\left|g_{L L}^{S}\right|^{2}+\left|g_{L R}^{S}\right|^{2}+\left|g_{R L}^{S}\right|^{2}+\left|g_{R R}^{S}\right|^{2}\right) \\
& +\left(\left|g_{L L}^{V}\right|^{2}+\left|g_{L R}^{V}\right|^{2}+\left|g_{R L}^{V}\right|^{2}+\left|g_{R R}^{V}\right|^{2}\right)+3\left(\left|g_{L R}^{T}\right|^{2}+\left|g_{R L}^{T}\right|^{2}\right)=1 .
\end{aligned}
$$

This restricts the allowed ranges of the coupling constants to $\left|g^{S}\right| \leq 2,\left|g^{V}\right| \leq 1$, and $\left|g^{T}\right| \leq \frac{1}{\sqrt{3}}$. The overall normalization can be incorporated into $G_{0}$ which then accounts for deviations from the Fermi coupling constant $G_{\text {Fermi }}$. We have to note that the precision of the muon life time measurement is much higher than the one obtained for definition of $G_{\text {Fermi }}$ via other parameters of the SM, i.e. $\alpha_{\mathrm{QED}}, M_{Z}, M_{W}$ etc. Unfortunately, for this reason the extremely precise measurement of $G_{\text {Fermi }}$ does not provide any valuable test of models beyond the SM.

In this paper we provide a parameterization of radiative muon (tau) decays in terms of the generalized Michel parameters [9, 10] which are certain bilinear combinations of the coupling constants $g_{\epsilon \omega}^{\gamma}$. Our aim is to take into account the exact dependence on the charged fermion masses.

\section{Radiative muon decay}

At the Born level the decay

$$
\mu^{-}\left(p_{\mu}\right) \rightarrow e^{-}\left(p_{e}\right)+\nu_{\mu}\left(k_{1}\right)+\bar{\nu}_{e}\left(k_{2}\right)+\gamma\left(p_{\gamma}\right)
$$


was considered by many authors since late fifties $[9,18,19]$. Here we follow the notation adapted in the review [1] and represent the differential width of this decay in the form

$$
\begin{aligned}
\frac{d \Gamma\left(\mu^{ \pm} \rightarrow e^{ \pm} \bar{\nu} \nu \gamma\right)}{d x d y d \Omega_{e} d \Omega_{\gamma}} & =\Gamma_{0} \frac{\alpha_{\mathrm{QED}}}{64 \pi^{3}} \frac{\beta_{e}}{y}\left[F(x, y, d) \mp \beta_{e} P_{\mu} \cos \theta_{e} G(x, y, d) \mp P_{\mu} \cos \theta_{\gamma} H(x, y, d)\right], \\
\Gamma_{0} & =\frac{G_{\mathrm{Fermi}}^{2} m_{\mu}^{5}}{192 \pi^{3}}, \quad d=1-\beta_{e} \cos \theta_{e \gamma}, \quad \beta_{e}=\sqrt{1-\frac{m_{e}^{2}}{E_{e}^{2}}},
\end{aligned}
$$

where $\Omega_{e, \gamma}$ are solid angles of the observable final state particles; $\theta_{e}$ and $\theta_{\gamma}$ are the angles between the muon spin and the electron and photon momenta, respectively; $P_{\mu}$ is the muon polarization degree; $\theta_{e \gamma}$ is the angle between the electron and photon momenta; $x$ and $y$ are energy fractions of electron and photon, respectively, $x \equiv 2 E_{e} / m_{\mu}$ and $y \equiv 2 E_{\gamma} / m_{\mu}$.

Functions $F, G$, and $H$ are polynomials in the electron to muon mass ratio:

$$
\mathcal{F}(x, y, d)=\sum_{k=1}^{5}\left(\frac{m_{e}}{m_{\mu}}\right)^{k} \mathcal{F}^{(k)}, \quad \mathcal{F} \equiv F, G, H .
$$

We computed these functions in the tree-level approximation. Analytical calculations were performed with the help of the FORM computer language [20]. The explicit expression for these functions are given in appendix A below. These functions depend on the generalized Michel parameters $\rho, \eta, \bar{\eta}, \xi, \delta, \kappa, \alpha$, and $\beta$, see appendix B. The dependence on $\bar{\eta}, \kappa, \alpha$, and $\beta$ provides important additional information about the structure of weak interactions with respect to the studies of non-radiative muon and tau decays, see [21]. Note that the dependence on $\alpha$ and $\beta$ appear only in terms suppressed by the first power of the mass ratio $r \equiv m_{e} / m_{\mu} \approx 5 \cdot 10^{-3}$ (or $m_{\mu} / m_{\tau} \approx 6 \cdot 10^{-2}$ for the $\tau \rightarrow \mu \nu \bar{\nu}$ decay). The presence of the contributions proportional to the first power of the mass ratio (and in general in odd powers) is a non trivial effect. Presumably, it is related to a hidden spin flip. An analogous effect takes place in the one-loop corrections to polarized muon decay spectrum: expansion of the exact result [22] for these corrections contains some terms which are linear in the electron to muon mass ratio.

The contributions suppressed by the electron to muon mass ratio can be important for modern high-precision experiments on muon and especially tau lepton radiative decays. As one can see from (A.1), (A.2), and (A.3), the most important higher order effect is given by function $F^{(1)}(x, y, d)$ since it is the only one being linear in the charged lepton mass ratio. Moreover, this function depends on the generalized Michel parameters $\alpha$ and $\beta$ which do not appear in the lowest approximation in $r$. In addition, this function contains terms proportional to $d^{-1}$ which provide a considerable enhancement in the kinematical domain of collinear radiation of photons along the electron direction of motion. Thus one can hope that future experiments on radiative muon and tau decays can provide additional information on the Michel parameter values.

We verified our results by comparison with the ones existing in the literature. We have checked that in the case of $V-A$ interactions our results completely agree with the formulae given in [1]. For the case of general interactions in the limit $m_{e} \ll m_{\mu}$, we performed a comparison with the results of [18]. Here the agreement is not complete. 
Namely, we have the coincidence for functions $\left.F(x, y, d)\right|_{m_{e} \rightarrow 0}$ and $\left.G(x, y, d)\right|_{m_{e} \rightarrow 0}$. But we encountered a small discrepancy for $\left.H(x, y, d)\right|_{m_{e} \rightarrow 0}$, which can be removed after the following modification in one term in the appendix of [18]:

$$
F_{1}^{T \gamma}=-2\left[x^{2} y^{2}(1-y)+2 x^{3} y\right] \rightarrow F_{1}^{T \gamma}=-2\left[x^{2} y^{2}(1-y)+2 x^{3} y^{2}\right] .
$$

Moreover, one more obvious misprint in [18] has to be corrected in eq. (4b):

$$
\left[\Delta+\left(2 / \mu^{2} x^{2}\right)\right] \rightarrow\left[\Delta+\left(2 m_{e}^{2} / \mu^{2} x^{2}\right)\right]
$$

Note that the expression in square brackets above is a certain approximation of quantity $d$ given in (3.2).

The NLO QED radiative corrections to this process (for the case of the pure $V-A$ interactions) were recently calculated in [23] with taking into account the exact dependence on the final state charged lepton mass. Earlier, the one-loop corrections to radiative muon decay were also considered in $[24,25]$. Besides the QED effects, radiative corrections to the $W$ boson propagator within the Standard Model become numerically relevant [26]. The processes of five-body leptonic decays of muons and tau leptons can be also used to get an additional information about the structure of weak interactions, see e.g. [27].

\section{Conclusions}

Our results can be used in the analysis of high-precision experimental data on $\tau$ and muon radiative decays in order to extract the generalized Michel parameters. The exact dependence on the final charged lepton mass is taken into account. The corresponding effect for certain cases is linear in the ratio of the final and initial charged lepton masses. So it becomes numerically important first of all for studies of the $\tau \rightarrow \mu \bar{\nu} \nu \gamma$ decay. A correction to the result of paper [18] is also made.

\section{Acknowledgments}

We are grateful to D. Epifanov and S. Eidelman for statement of the problem and useful discussions and to E. Akzhigitova for the help with cross checks of the results.

\section{A Functions $F, G$, and $H$}

Here we give the explicit formulae for functions $F, G$, and $H$ which appear in (3.2):

$$
\begin{aligned}
F^{(0)}= & 32 \rho\left(\frac{16 x^{2} y+12 x y^{2}+4 y^{3}+8 x^{3}-3 y^{2}-6 x^{2}-6 x y}{3 d}-\frac{10}{3} x^{2} y^{2}-\frac{8 x^{3} y}{3}-2 x y^{3}\right. \\
& \left.-\frac{x y^{2}}{3}-\frac{5 x^{2} y}{3}-\frac{4 x^{3}}{3}+x y+x^{2}+x y d\left(\frac{4 x^{2}}{3}+x^{2} y+x y^{2}+\frac{3}{4} x y-\frac{x}{2}\right)-\frac{x^{3} y^{2} d^{2}}{6}(2+y)\right) \\
& +\bar{\eta} 8 x y^{2}(2 y+4 x-x d-x y d-2)+48 \frac{y^{2}+2 x y+2 x^{2}-y^{3}-2 x^{3}-3 x y^{2}-4 x^{2} y}{d}
\end{aligned}
$$




$$
\begin{aligned}
& +96 x^{3} y+112 x^{2} y^{2}+56 x y^{3}+48 x^{2}(x+y-1)+16 x y^{2}-48 x y \\
& +4 x y d\left(6 x-7 x y(1+y)-12 x^{2}-9 x^{2} y\right)+6 x^{3} y^{2} d^{2}(2+y), \\
& F^{(1)}=\beta\left(\frac{24 x(x+y-1)}{d}-\frac{4 y^{2}}{d}+4 x y^{2}-12 x(x y+x+y-1)-x^{2} y^{2} d+6 x^{2} y d\right) \\
& +\alpha\left(\frac{12 x(1-x-y)}{d}+6 x(x y+x+y-1)-3 x^{2} y d\right), \\
& F^{(2)}=16 \rho\left(\frac{8(x+y)(3-4 x-4 y)}{3 x d^{2}}+\frac{18 y^{2}-12 x^{2}+20 x y-16 x-12 y}{3 d}\right. \\
& \left.+2 x^{2} y-\frac{2 x y^{2}}{3}+2 x^{2}+2 x y+\frac{8 x}{3}-\frac{x^{2} y d(2+y)}{2}\right)+16 \bar{\eta}\left(-x y^{2}-\frac{2 y^{2}}{d}\right) \\
& +\frac{192\left(x^{2}+y^{2}+2 x y-x-y\right)}{x d^{2}}+\frac{96 x^{2}-112 y^{2}-96 x y+96 y}{d}-48 x^{2} y+16 x y^{2} \\
& -48 x^{2}-48 x y+12 x^{2} y d(2+y), \\
& F^{(3)}=96 \eta\left(4 \frac{x+y-1}{x d^{2}}+\frac{2(x-y)}{d}-x\right) \text {, } \\
& F^{(4)}=64 \rho \frac{8+6 x+6 y-3 x y d}{3 x d^{2}}+96 \frac{x y d-2 y-2 x}{x d^{2}}, \\
& F^{(5)}=\eta\left(-\frac{384}{x d^{2}}\right) \\
& G^{(0)}=8 \xi\left(\frac{4 x^{3}+6 x^{2} y-4 x^{2}-2 x y}{d}+\frac{4 x y^{2}}{3 d}-3 x^{3} y-\frac{5}{3} x^{2} y^{2}-2 x^{3}-x^{2} y+2 x^{2}\right. \\
& \left.+\frac{x^{3} y d(2+y)}{2}\right)+32 \delta \xi\left(\frac{x y}{d}-\frac{10 x y^{2}}{9 d}+\frac{2 x^{2}}{d}-\frac{4 x^{2} y}{d}-\frac{8 x^{3}}{3 d}-x^{2}+\frac{5 x^{2} y}{6}\right. \\
& \left.+\frac{11 x^{2} y^{2}}{9}+\frac{4 x^{3}}{3}+2 x^{3} y-\frac{x^{3} y d}{3}(2+y)\right)+8 \kappa \xi x y^{2}\left(x-\frac{2}{d}\right), \\
& G^{(2)}=8 \xi\left(\frac{8(1-x-y)}{d^{2}}+\frac{2 x y}{d}-\frac{4 x^{2}}{d}+2 x^{2}+x^{2} y\right) \\
& +16 \delta \xi\left(\frac{8(4 x+4 y-3)}{3 d^{2}}-\frac{2 x y}{d}+\frac{20 x^{2}}{3 d}-\frac{10 x^{2}}{3}-\frac{5 x^{2} y}{3}\right), \\
& G^{(4)}=\xi \frac{64}{d^{2}}-\delta \xi \frac{640}{3 d^{2}}, \\
& G^{(1)}=0, \quad G^{(3)}=0, \quad G^{(5)}=0, \\
& H^{0}=8 \xi\left(\frac{2 y\left(x^{2}+y^{2}-y-x\right)}{d}+\frac{14 x y^{2}}{3 d}-\frac{7}{3} x y^{3}-x^{3} y+\frac{2 x y^{2}}{3}-3 x^{2} y^{2}+x^{2} y\right. \\
& \left.+\frac{7}{6} x^{2} y^{3} d+x^{3} y^{2} d-\frac{x^{2} y^{2} d}{2}-\frac{x^{3} y^{3} d^{2}}{4}\right)+8 \delta \xi\left(\frac{4 y\left(3 x+3 y-4 x^{2}-4 y^{2}\right)}{3 d}-\frac{104 x y^{2}}{9 d}\right.
\end{aligned}
$$




$$
\begin{aligned}
& \left.+\frac{40}{9} x y^{3}+\frac{8 x^{3} y}{3}+\frac{20}{3} x^{2} y^{2}+\frac{4 x y^{2}}{9}-2 x^{2} y+x^{2} y^{2} d-\frac{20}{9} x^{2} y^{3} d-\frac{8}{3} x^{3} y^{2} d+\frac{2}{3} x^{3} y^{3} d^{2}\right) \\
& +8 \kappa \xi\left(\frac{2 x y^{2}}{d}+2 x y^{3}+3 x^{2} y^{2}-4 x y^{2}-x^{2} y^{3} d\right), \\
H^{(2)}= & 8 \xi\left(\frac{8 y(1-y-x)}{x d^{2}}+\frac{14 y^{2}}{3 d}+\frac{2 x y}{d}-\frac{4 y}{d}-\frac{2 x y^{2}}{3}+x^{2} y-\frac{x^{2} y^{2} d}{2}\right) \\
& +8 \delta \xi\left(\frac{16 y(4 y+4 x-3)}{3 x d^{2}}-\frac{116 y^{2}}{9 d}-\frac{4 x y}{d}+\frac{8 y}{d}+\frac{20 x y^{2}}{9}-\frac{10 x^{2} y}{3}+\frac{5 x^{2} y^{2} d}{3}\right) \\
& +16 \kappa \xi\left(-x y^{2}-\frac{2 y^{2}}{d}\right), \quad \\
H^{(4)}= & 32 \xi\left(\frac{2 y}{x d^{2}}-\frac{y}{d}\right)+320 \delta \xi\left(\frac{y}{3 d}-\frac{2 y}{3 x d^{2}}\right), \\
H^{(1)}= & 0, \quad H^{(3)}=0, \quad H^{(5)}=0 .
\end{aligned}
$$

\section{B Generalized Michel parameters}

For the sake of completeness, we give here the explicit expressions for the generalized Michel parameters defined by Kinoshita and Sirlin [9, 10], see also [17] for details, via the coupling constants $g_{\epsilon \omega}^{\gamma}$ which enter $(2.1)$ :

$$
\begin{aligned}
\rho & =\frac{3 b+6 c}{16}=\frac{3}{4}+\frac{3}{4} \cdot \frac{(2 c-a)}{16}, \quad \eta=\frac{\alpha-2 \beta}{16}, \quad \bar{\eta}=\frac{a+2 c}{16}, \\
\kappa \xi & =\frac{a_{1}+2 c_{1}}{16}, \quad \delta \xi=\frac{6 c_{1}-3 b_{1}}{16}, \quad \xi=\frac{14 c_{1}-3 a_{1}-4 b_{1}}{16}, \\
\alpha & =8 \operatorname{Re}\left(g_{R L}^{V}\left(g_{L R}^{S *}+6 g_{L R}^{T *}\right)+g_{L R}^{V}\left(g_{R L}^{S *}+6 g_{R L}^{T *}\right)\right), \quad \beta=-4 \operatorname{Re}\left(g_{R R}^{V} g_{L L}^{S *}+g_{L L}^{V} g_{R R}^{S *}\right), \\
a & =16\left(\left|g_{R L}^{V}\right|^{2}+\left|g_{L R}^{V}\right|^{2}\right)+\left|g_{R L}^{S}+6 g_{R L}^{T}\right|^{2}+\left|g_{L R}^{S}+6 g_{L R}^{T}\right|^{2}, \\
b & =4\left(\left|g_{R R}^{V}\right|^{2}+\left|g_{L L}^{V}\right|^{2}\right)+\left|g_{R R}^{S}\right|^{2}+\left|g_{L L}^{S}\right|^{2}, \\
c & =\frac{1}{2}\left|g_{R L}^{S}-2 g_{R L}^{T}\right|^{2}+\frac{1}{2}\left|g_{L R}^{S}-2 g_{L R}^{T}\right|^{2}, \\
a_{1} & =16\left(\left|g_{R L}^{V}\right|^{2}-\left|g_{L R}^{V}\right|^{2}\right)+\left|g_{R L}^{S}+6 g_{R L}^{T}\right|^{2}-\left|g_{L R}^{S}+6 g_{L R}^{T}\right|^{2}, \\
b_{1} & =4\left(\left|g_{R R}^{V}\right|^{2}-\left|g_{L L}^{V}\right|^{2}\right)+\left|g_{R R}^{S}\right|^{2}-\left|g_{L L}^{S}\right|^{2}, \\
c_{1} & =\frac{1}{2}\left|g_{R L}^{S}-2 g_{R L}^{T}\right|^{2}-\frac{1}{2}\left|g_{L R}^{S}-2 g_{L R}^{T}\right|^{2} .
\end{aligned}
$$

Note that we used above the normalization condition (2.3), which can be re-written also as

$$
a+4 b+6 c=16 .
$$

In the limit of the pure $V-A$ interactions we have $\rho=\delta=\frac{3}{4}, \xi=1, \eta=\bar{\eta}=\kappa=\alpha=\beta=0$. 
Open Access. This article is distributed under the terms of the Creative Commons Attribution License (CC-BY 4.0), which permits any use, distribution and reproduction in any medium, provided the original author(s) and source are credited.

\section{References}

[1] Y. Kuno and Y. Okada, Muon decay and physics beyond the standard model, Rev. Mod. Phys. 73 (2001) 151 [hep-ph/9909265] [INSPIRE].

[2] A. Pich and J.P. Silva, Constraining new interactions with leptonic $\tau$ decays, Phys. Rev. D 52 (1995) 4006 [hep-ph/9505327] [INSPIRE].

[3] A. Rouge, Tau lepton Michel parameters and new physics, Eur. Phys. J. C 18 (2001) 491 [hep-ph/0010005] [INSPIRE].

[4] A. Celis, V. Cirigliano and E. Passemar, Model-discriminating power of lepton flavor violating $\tau$ decays, Phys. Rev. D 89 (2014) 095014 [arXiv: 1403.5781] [INSPIRE].

[5] A. Hayreter and G. Valencia, Spin correlations and new physics in $\tau$-lepton decays at the LHC, JHEP 07 (2015) 174 [arXiv:1505.02176] [INSPIRE].

[6] S. Eidelman, D. Epifanov, M. Fael, L. Mercolli and M. Passera, $\tau$ dipole moments via radiative leptonic $\tau$ decays, JHEP 03 (2016) 140 [arXiv:1601.07987] [INSPIRE].

[7] BABAR collaboration, J.P. Lees et al., Measurement of the branching fractions of the radiative leptonic $\tau$ decays $\tau \rightarrow e \gamma \nu \bar{\nu}$ and $\tau \rightarrow \mu \gamma \nu \bar{\nu}$ at BABAR, Phys. Rev. D 91 (2015) 051103 [arXiv: 1502.01784] [INSPIRE].

[8] L. Michel, Interaction between four half spin particles and the decay of the $\mu$ meson, Proc. Phys. Soc. A 63 (1950) 514 [inSPIRE].

[9] T. Kinoshita and A. Sirlin, Muon Decay with Parity Nonconserving Interactions and Radiative Corrections in the Two-Component Theory, Phys. Rev. 107 (1957) 593 [InSPIRE].

[10] T. Kinoshita and A. Sirlin, Polarization of Electrons in Muon Decay with General Parity-Nonconserving Interactions, Phys. Rev. 108 (1957) 844 [INSPIRE].

[11] ARGUS collaboration, H. Albrecht et al., Determination of the Michel parameter in tau decay, Phys. Lett. B 246 (1990) 278 [inSPIRE].

[12] OPAL collaboration, K. Ackerstaff et al., Measurement of the Michel parameters in leptonic tau decays, Eur. Phys. J. C 8 (1999) 3 [hep-ex/9808016] [INSPIRE].

[13] CLEO collaboration, R. Ammar et al., A Measurement of the Michel parameters in leptonic decays of the tau, Phys. Rev. Lett. 78 (1997) 4686 [INSPIRE].

[14] ALEPH collaboration, A. Heister et al., Measurement of the Michel parameters and the $\nu_{\tau}$ helicity in $\tau$ lepton decays, Eur. Phys. J. C 22 (2001) 217 [INSPIRE].

[15] Belle collaboration, A. Abdesselam et al., Study of Michel parameters in leptonic $\tau$ decays at Belle, arXiv:1409.4969 [INSPIRE].

[16] W. Fetscher, H.J. Gerber and K.F. Johnson, Muon Decay: Complete Determination of the Interaction and Comparison with the Standard Model, Phys. Lett. B 173 (1986) 102 [INSPIRE].

[17] Particle Data Group collaboration, K.A. Olive et al., Review of Particle Physics, Chin. Phys. C 38 (2014) 090001 [InSPIRE]. 
[18] C. Fronsdal and H. Uberall, $\mu$-Meson Decay with Inner Bremsstrahlung, Phys. Rev. 113 (1959) 654 [InSPIRE].

[19] S.G. Eckstein and R.H. Pratt, Radiative muon decay, Ann. Phys. 8 (1959) 297.

[20] J.A.M. Vermaseren, New features of FORM, math-ph/0010025 [INSPIRE].

[21] W. Eichenberger, R. Engfer and A. Van Der Schaaf, Measurement of the parameter $\bar{\eta}$ in the radiative decay of the muon as a test of the v-a structure of the weak interaction, Nucl. Phys. A 412 (1984) 523 [INSPIRE].

[22] A.B. Arbuzov, First order radiative corrections to polarized muon decay spectrum, Phys. Lett. B 524 (2002) 99 [Erratum ibid. B 535 (2002) 378] [hep-ph/0110047] [INSPIRE].

[23] M. Fael, L. Mercolli and M. Passera, Radiative $\mu$ and $\tau$ leptonic decays at NLO, JHEP 07 (2015) 153 [arXiv: 1506.03416] [INSPIRE].

[24] A. Fischer, T. Kurosu and F. Savatier, QED one loop correction to radiative muon decay, Phys. Rev. D 49 (1994) 3426 [INSPIRE].

[25] A.B. Arbuzov and E.S. Scherbakova, One loop corrections to radiative muon decay, Phys. Lett. B 597 (2004) 285 [hep-ph/0404094] [INSPIRE].

[26] M. Fael, L. Mercolli and M. Passera, W-propagator corrections to $\mu$ and $\tau$ leptonic decays, Phys. Rev. D 88 (2013) 093011 [arXiv: 1310.1081] [InSPIRE].

[27] A. Flores-Tlalpa, G. López Castro and P. Roig, Five-body leptonic decays of muon and tau leptons, JHEP 04 (2016) 185 [arXiv: 1508.01822] [INSPIRE]. 